\title{
Charting the Transient Radio Sky on Sub-Second Time-Scales with LOFAR
}

\author{
J. W. T. Hessels ${ }^{1,2}$ (and the LOFAR Transients Key Science Project) \\ ${ }^{1}$ Netherlands Institute for Radio Astronomy (ASTRON), 7990 AA Dwingeloo, \\ The Netherlands \\ ${ }^{2}$ Astronomical Institute "Anton Pannekoek," University of Amsterdam, The Netherlands \\ email: J.W.T.Hessels@uva.nl
}

Summary. The LOw Frequency ARray (LOFAR) is a radio interferometric telescope that promises to open a largely unexplored window on transient sources in the "radio sky", from time-scales of nanoseconds to years. An important aspect of this will be the study of radioemitting neutron stars in their various incarnations: slow pulsars, young pulsars, millisecond pulsars, magnetars, rotating radio transients, intermittent pulsars, et cetera. Pulsars and their brethren are the prototype of the more general "fast transients": sub-second, dispersed radio bursts which point the way to extreme, and potentially still unknown, phenomena. For instance, prompt radio bursts from supernovæ and other extra-galactic bursts have been hypothesized; these could prove to be powerful cosmological probes.

This talk discussed LOFAR's impressive ability to observe pulsars and to enlarge greatly the discovery space for (even rarer) fast transients. It also presented the latest pulsar observations made during LOFAR's commissioning period. These are demonstrating powerful observing techniques that will be crucial for the next generation of radio telescopes as well as the effort to increase our understanding of the dynamic nature of the Universe.

An expanded version of the talk can be found at http://adsabs.harvard.edu/abs/2011A 